\title{
Die Sinnfrage in der Medizinethik
}

\author{
Roland Kipke $\cdot$ Markus Rüther
}

Angenommen: 28. September 2021

(C) Der/die Autor(en) 2021

Der Sinn des Lebens - gewiss ein Thema für manche Klinikseelsorgerin, aber nicht für eine säkulare, wissenschaftliche Medizinethik? So mag es auf den ersten Blick erscheinen, vor allem, wenn man „Sinn“ in traditioneller Weise als einen vorgegebenen Zweck des Daseins versteht, was heute fast nur noch in einer religiösen Perspektive möglich zu sein scheint. Die neuere philosophische Debatte um ,meaning in life" dreht sich allerdings weniger um diese metaphysische Dimension der Sinnfrage, sondern verfolgt einen bescheideneren Anspruch: Sinn wird hier als Teil eines individuellen menschlichen Lebens verstanden, genauer: als eine eigenständige Dimension des guten Lebens, die sich vom Glück und - zumindest teilweise von Moralität unterscheidet. ${ }^{1}$ Und das heißt: Sinn ist nicht Thema allein für Grübler und Schöngeister, sondern er ist für alle Menschen existentiell bedeutsam.

\footnotetext{
1 Seit gut zwei Jahrzehnten heben die Teilnehmer dieser internationalen Debatte die Frage nach dem Sinn nicht nur neu aufs Tapet, sondern stellen neue Fragen und geben neue und weiterführende Antworten. Hierzu gehören Autorinnen wie John Cottingham, Iddo Landau, Thaddeus Metz und Susan Wolf. Und auch in Deutschland finden sich erste Ansätze für eine solche Debatte. Zu nennen sind etwa die Arbeiten von Holmer Steinfath und Ursula Wolf, die das Sinnthema in ihrer Auseinandersetzung mit dem guten Leben berücksichtigen. Im Vergleich zur angelsächsischen Debatte wird es jedoch meistens am Rande behandelt. Die Ausnahme von der Regel bilden allenfalls die Beiträge im Schwerpunkt von Kühler et al. (2018) und einige Beiträge, die eine explizite Position in der Debatte verteidigen. Siehe Siep (2013) und die Weiterentwicklungen in Siep (2020), Kap. 6 sowie Kipke (2014) und Rüther (i. V.). Vgl. für diese Diagnose samt eines umfangreichen Überblicks über die Meaning-in-Life-Debatte Rüther (2021a) und Rüther (2021b).
}

Dr. Roland Kipke $(\bowtie)$

Abteilung Philosophie, Universität Bielefeld, 33501 Bielefeld, Deutschland

E-Mail: r.kipke@uni-bielefeld.de

Dr. phil. Markus Rüther

Institut für Neurowissenschaften und Medizin, Ethik in den Neurowissenschaften (INM-8),

Forschungszentrum Jülich GmbH, 52425 Jülich, Deutschland

E-Mail: m.ruether@fz-juelich.de 
Wenn Sinn wesentlicher Teil eines guten Lebens ist, dürfte er auch für medizinethische Fragestellungen von Bedeutung sein. Am augenfälligsten ist das vielleicht bei Entscheidungen am Lebensende: Welche Rolle spielen dabei Annahmen über den Sinn des noch zu erwartenden Lebens? Und welche Rolle sollten sie spielen - aus Patientenperspektive und aus Sicht der behandelnden Ärzte? Wie verändert eine Demenzdiagnose den Blick auf den Sinn des eigenen Lebens? Wie verändert überhaupt die Erfahrung von Krankheit das eigene Sinnverständnis? Und sind nicht speziell psychische Erkrankungen als Sinnkrisen oder Einschränkungen des Sinnentfaltungspotentials zu verstehen? Wenn ja, müsste dann die Psychiatrie ihr übergeordnetes Therapieziel nicht gerade in der Befähigung zur Führung eines sinnvollen Lebens sehen $?^{2}$ Ebenso drängen sich Sinnfragen bei anderen Praxisfeldern auf, wie etwa bei einer biomedizinisch bewirkten Verlängerung der menschlichen Lebensdauer oder bei der Gestaltung der eigenen Persönlichkeit durch Neuroenhancement.

So verschieden die Problemlagen auch sind, führt das Nachdenken über sie doch immer wieder zu gemeinsamen Grundfragen: Welches Verständnis eines sinnvollen Lebens liegt Entscheidungen und entscheidungsrelevanten Überzeugungen zugrunde? Inwiefern lässt es sich rechtfertigen? Gibt es überhaupt universalisierbare, also für alle verbindliche Kriterien für Sinnhaftigkeit oder variieren diese von Person zu Person? Wenn es universalisierbare Kriterien gibt, wie lassen sich diese begründen? Und selbst wenn es für alle gültige Kriterien gibt, dürfen diese zur Grundlage von allgemeingültigen Regeln oder gar von politischen Entscheidungen gemacht werden? Kurzum, es geht sowohl um die deskriptive Analyse vorhandener - und zumeist impliziter - sinntheoretischer Annahmen als auch um die normative Frage, welche Rolle der Lebenssinn in Medizin und Medizinethik spielen darf und soll.

Diesen Fragen nachzugehen und die Bedeutung der Sinnfrage für die Medizinethik freizulegen ist das Anliegen dieses Heftes. Die explizite und systematische Behandlung der Sinnthematik kann neues Licht auf Probleme werfen, die heute als theoretisch erschöpft gelten, sowie neue Perspektiven für ihre Lösung entwickeln.

Damit verknüpft das Themenheft zwei im deutschsprachigen Bereich bislang weitgehend voneinander isolierte Debattenstränge miteinander - die Frage nach dem sinnvollen Leben einerseits und die medizinethischen Fragen andererseits. Zugleich macht es eine bislang vorwiegend international geführte Diskussion für das deutschsprachige medizinethische Denken fruchtbar. Der Fokus des Heftes liegt also nicht in einem einzelnen medizinischen Praxisfeld, sondern in einer bestimmten Wertdimension des guten Lebens, die sich - so die leitende These - mehr oder weniger durch alle medizinethischen Themenfelder zieht.

Den Auftakt des Themenschwerpunkts macht der Überblicksartikel von Thaddeus Metz. Metz hat die internationale Meaning-in-Life-Debatte in den letzten zwei Jahrzehnten durch seine zahlreichen Forschungsarbeiten maßgeblich mitgeprägt. In seinem Beitrag in diesem Heft klärt er die Kategorie des Sinns, grenzt sie von anderen ethischen Dimensionen ab und skizziert, inwiefern sie für verschiedene bioethische Problemfelder relevant ist - von der Reproduktionsmedizin über Fragen der Lebensverlängerung bis hin zu Entscheidungen am Lebensende.

\footnotetext{
2 Vgl. Metz (2016).
} 
Für viele Menschen stellt sich die Frage nach dem Sinn, wenn sie schwer erkranken. Oft wird vom „Sinn einer Krankheit“ gesprochen. Doch ist diese Redeweise überhaupt gerechtfertigt? Und wenn ja, in welchem Sinne? Ralf Stoecker kartiert in seinem Beitrag das Bedeutungsfeld von „Krankheit und Sinn“. Dabei macht er nicht nur klar, welche unterschiedlichen Bedeutungen der Begriff „Sinn“ in Bezug auf Krankheit annehmen kann, sondern auch, wann diese Redeweise sinnvoll ist und wann nicht.

Nach diesen zwei Artikeln mit übergeordneten Fragestellungen folgen Beiträge, die sich einzelnen medizinethischen Konfliktfeldern widmen. Markus Rüther nimmt sich der Debatte um das sogenannte Human Enhancement an. Genauer gesagt: Er untersucht die Frage, ob Praktiken des Enhancements notwendig zu einem Verlust an Lebenssinn führen. In seiner Konklusion kommt Rüther zu dem Ergebnis, dass diese Sorge weitgehend unbegründet ist, da die befürchteten negativen Konsequenzen für den Sinn wenig wahrscheinlich sind oder sich leicht vermeiden lassen.

In besonderem Maße drängt sich die Frage nach dem Sinn eines Lebens auf, wenn das Leben als ganzes in Frage steht. So verwundert es nicht, dass drei Beiträge ihren Blick auf die letzte Lebensphase und das Lebensende richten. Das tun sie allerdings auf ganz unterschiedliche Weise. Susanne Hiekel schlägt vor, den Schrecken, den die Diagnose Demenz bei Betroffenen auslösen kann, auch als Angst vor Sinnverlust zu verstehen. In Ihrem Artikel überprüft sie, ob diese Angst berechtigt ist, und legt dazu die einflussreiche Sinntheorie von Susan Wolf zugrunde. Das Ergebnis von Hiekels Untersuchung hat das Potenzial, der Demenzdiagnose etwas von ihrem Schrecken zu nehmen: Bis zu einer mittelschweren Demenz ist es durchaus noch möglich, ein sinnvolles Leben zu führen. Bei Patienten mit schwerer Demenz sei das zwar nicht mehr möglich, sie würden dies aber ohnehin nicht mehr als Verlust erleben.

In dem Beitrag von Sebastian Muders steht das Lebensende nicht im Mittelpunkt, aber der konkrete Fall eines assistierten Suizids bildet den Ausgangspunkt und Prüfstein für seine Untersuchung praktischer Gründe, die sich auf den Sinn im Leben beziehen. Muders dreifache These lautet, dass solche Sinn-Gründe Gründe eigener Art sind, gerade auch gegenüber Gründen, die sich auf die Würde beziehen; dass Sinn-Gründe und Würde-Gründe dennoch einige strukturelle Gemeinsamkeiten aufweisen; und dass Sinn-Gründen gerade auch bei Entscheidungen am Lebensende das moralische Primat gegenüber Würde-Gründen zukommt.

Auch Roland Kipke geht von einer moralischen Relevanz des Sinns aus. Er widmet sich in seinem Beitrag ganz dem assistierten Suizid und sieht die Verneinung von Lebenssinn, die sich in Suiziden häufig ausspricht, nicht nur als eine individuelle, sondern als gesellschaftliche Angelegenheit. Kipkes These lautet, dass in dieser Interpretation von Suiziden der Schlüssel dazu liegt, nicht nur die medizinethische Frontstellung in dieser Frage zu überwinden, sondern auch einige verbreitete ethische Annahmen zu rechtfertigen.

Funding Open Access funding enabled and organized by Projekt DEAL. 
Open Access Dieser Artikel wird unter der Creative Commons Namensnennung 4.0 International Lizenz veröffentlicht, welche die Nutzung, Vervielfältigung, Bearbeitung, Verbreitung und Wiedergabe in jeglichem Medium und Format erlaubt, sofern Sie den/die ursprünglichen Autor(en) und die Quelle ordnungsgemäß nennen, einen Link zur Creative Commons Lizenz beifügen und angeben, ob Änderungen vorgenommen wurden.

Die in diesem Artikel enthaltenen Bilder und sonstiges Drittmaterial unterliegen ebenfalls der genannten Creative Commons Lizenz, sofern sich aus der Abbildungslegende nichts anderes ergibt. Sofern das betreffende Material nicht unter der genannten Creative Commons Lizenz steht und die betreffende Handlung nicht nach gesetzlichen Vorschriften erlaubt ist, ist für die oben aufgeführten Weiterverwendungen des Materials die Einwilligung des jeweiligen Rechteinhabers einzuholen.

Weitere Details zur Lizenz entnehmen Sie bitte der Lizenzinformation auf http://creativecommons.org/ licenses/by/4.0/deed.de.

Interessenkonflikt R. Kipke und M. Rüther geben an, dass kein Interessenkonflikt besteht.

\section{Literatur}

Kipke R (2014) Der Sinn des Lebens und das gute Leben. Z Philosoph Forsch 68(2):180-202

Kühler M, Muders S, Rüther M (2018) Schwerpunkt: Das Schöne, Wahre und Gute - Die neue Diskussion um das sinnvolle Leben. Z Prakt Philos 5(2):41-282

Metz T (2016) The proper aim of therapy: subjective well-being, objective goodness, or a meaningful life? In: Russo-Netzer P, Schulenberg SE, Batthyany A (Hrsg) Clinical perspectives on meaning. Positive and existential psychotherapy. Springer, Cham, S 17-35

Rüther M (i. V.) Was macht das Leben sinnvoll? Grundlinien einer normativ-inhaltlichen Theorie des sinnvollen Lebens. Unveröffentlichte Habilitationsschrift, Universität Bonn

Rüther M (2021a) Meaning in Life oder: Die Debatte um das sinnvolle Leben - Überblick über ein neues Forschungsthema in der analytischen Ethik. Teil 1: Grundlagen. Z Philos Forsch 75(1):115-155

Rüther M (2021b) Meaning in Life oder: Die Debatte um das sinnvolle Leben - Überblick über ein neues Forschungsthema in der analytischen Ethik. Teil 2: normativ-inhaltliche Fragen. Z Philos Forsch 75(2):316-354

Siep L (2013) Was für ein Leben? Was für ein Sinn? In: Hoesch M, Muders S, Rüther M (Hrsg) Glück - Werte - Sinn: Metaethische, ethische und theologische Zugänge zur Frage nach dem guten Leben. De Gruyter, Berlin New York, S 91-108

Siep L (2020) Technisierung der Natur. Historisierung der Moral. Ziele und Grenzen. Mentis, Münster 